\title{
Vertex function compliant with the Ward identity for quasiparticle self-consistent calculations beyond $G W$
}

\author{
Alexey Tal $\odot,{ }^{1, *}$ Wei Chen $\odot,{ }^{2}$ and Alfredo Pasquarello $\odot^{1}$ \\ ${ }^{1}$ Chaire de Simulation à l'Echelle Atomique (CSEA), Ecole Polytechnique Fédérale de Lausanne (EPFL), CH-1015 Lausanne, Switzerland \\ ${ }^{2}$ Institute of Condensed Matter and Nanoscience (IMCN), Université catholique de Louvain, B-1348 Louvain-la-Neuve, Belgium
}

(Received 10 July 2020; revised 3 December 2020; accepted 25 March 2021; published 8 April 2021)

\begin{abstract}
We extend the quasiparticle self-consistent approach beyond the $G W$ approximation by using a rangeseparated vertex function. The developed approach yields band gaps, dielectric constants, and band positions with an accuracy similar to highest-level electronic-structure calculations without exceeding the cost of regular quasiparticle self-consistent $G W$. We introduce an exchange-correlation kernel that accounts for the vertex over the full spatial range. In the long range it complies with the Ward identity, while it is approximated through the adiabatic local density functional in the short range. In this approach, the renormalization factor is balanced and the higher-order diagrams are effectively taken into account.
\end{abstract}

DOI: 10.1103/PhysRevB.103.L161104

Achieving an accurate description of the electronic structure of solids is a challenging problem in condensed matter physics. In particular, important properties of materials such as band gaps and excited states cannot be accurately reproduced within density-functional theory (DFT) [1-4]. A common approach to correct the calculated band gaps is Hedin's $G W$ approximation [5]. It has been shown that single-shot $G_{0} W_{0}$ corrections applied to DFT electronic structures improve the band gaps [6-8]. However, the outcome of this approach strongly depends on the starting point, i.e., the exchange-correlation (xc) functional [9-11]. The starting-point dependency can be overcome by solving Hedin's equations self-consistently, as in the quasiparticle self-consistent $G W$ (QS $G W$ ) scheme developed by van Schilfgaarde et al. [12,13]. However, straight QSGW overestimates the band gaps by $20 \%[12,14,15]$. van Schilfgaarde, Kotani, and Faleev argued that the electron-hole interaction included in the polarizability through the vertex function should address this issue $[12,13]$. Another drawback of QSGW is that it yields absolute band positions that are too deep for delocalized states and too shallow for localized states $[14,16,17]$. This is a highly relevant issue as the band positions play a determining role in photocatalysis, photovoltaics, and charge transport in materials.

The electron-hole interaction has been a long-standing problem in time-dependent density functional theory (TDDFT). This interaction gives rise to excitonic effects and is therefore highly important in optical spectra $[18,19]$. Since the random phase approximation (RPA) neglects these effects, they have to be included via the xc kernel $f_{\mathrm{xc}}$ [20]. The development of such kernels is an actively progressing field in which various approximations have been proposed $[19,20]$. The simplest one is the adiabatic local-density approximation

*alexey.tal@epfl.ch
(ALDA) [18]. It has been shown that ALDA vertex corrections in $G W$ calculations shift the absolute quasiparticle (QP) energies [21-23] but have very little effect on the band gaps [24]. In fact, ALDA lacks the correct asymptotic long-range (LR) behavior $1 / q^{2}$, which accounts for the electron-hole interaction [24].

In order to account for this interaction, the nanoquanta (NQ) kernel has been derived from the Bethe-Salpeter equation [25-27]. Notably, the NQ kernel has shown an excellent accuracy and established the state of the art for describing excitonic effects in optical spectra. Shishkin et al. [15] performed QSGW calculations with the NQ vertex in the polarizability achieving significant improvements in the band gaps and in the dielectric constants. However, the main drawback of the NQ kernel is its computational complexity, which makes it only applicable to small systems. An approximated xc kernel that is able to capture the excitonic effects has been developed by Sharma et al. [28]. By adopting this socalled bootstrap (Boot) approximation for the polarizability vertex, Chen and Pasquarello yielded band gaps of similar accuracy to NQ, but the ionization potentials (IPs) remained strongly overestimated [14]. Moreover, the Boot vertex lacks a formal derivation and has limited accuracy [28]. As Grüneis et al. showed, the consideration of the vertex function in the self-energy brings the IPs much closer to experiment [16]. However, this improvement comes at the demanding cost of including higher-order diagrams. Thus, it would be highly desirable to achieve such improvements through the development of accurate and reliable approximations for the vertex.

In the present Letter, we focus on efficient approximations to the vertex function in the QSGW method and demonstrate the potential of this approach. We represent the vertex function as a sum of a short-range and a long-range contribution. Such a range separation yields a vertex satisfying the renormalization balance and can be approximated more efficiently. Through the use of the Ward identity, we derive an expression for the long-range xc kernel that respects the charge conserva- 
tion and construct vertex corrections that effectively account for higher-order diagrams over the full spatial range. These vertex corrections yield an excellent accuracy for the band gaps and significantly improve the absolute band energies as well as the dielectric constants. Most importantly, this accuracy is achieved at practically no extra computational cost with respect to state-of-the-art QSGW .

In the many-body perturbation theory, the self-energy can be defined as $\Sigma=i G W \Gamma$ [5], where $G$ is the Green's function, $W$ the screened Coulomb potential, and $\Gamma$ the vertex function. The $G W$ approximation neglects the vertex by taking $\Gamma=1[5,7]$. Despite the lack of vertex corrections, non-selfconsistent $G_{0} W_{0}$ has been shown to give surprisingly good results [6-8]. The success of this approximation has been explained by the cancellation of the renormalization factor $Z$ [13]. A similar cancellation occurs in the self-consistent QSGW approach. We can write the Green's function as

$$
G=\frac{1}{\omega-H_{0}-\left[-V^{\mathrm{xc}}+\Sigma\left(\omega_{0}\right)+\left.\frac{\partial \Sigma}{\partial \omega}\right|_{\omega_{0}}\left(\omega-\omega_{0}\right)\right]+i \delta},
$$

where $H_{0}$ is a one-body Hamiltonian and $V^{\mathrm{xc}}$ is the nonlocal $\mathrm{xc}$ potential given by

$$
V^{\mathrm{xc}}=\frac{1}{2} \sum_{i j}\left|\psi_{i}\right\rangle\left\{\operatorname{Re}\left[\Sigma\left(\epsilon_{i}\right)\right]_{i j}+\operatorname{Re}\left[\Sigma\left(\epsilon_{j}\right)\right]_{i j}\right\}\left\langle\psi_{j}\right| .
$$

Considering that the noninteracting Green's function is defined as

$$
G_{0}=\frac{1}{\omega-H_{0}+i \delta},
$$

we can rewrite the Green's function as $G=Z G_{0}+\bar{G}$, where the first term represents the renormalized QP Green's function and the second term is an incoherent part neglected in QSGW [13]. The renormalization factor $Z$ accounts for the redistribution of the spectral weight from the main QP peak to the satellites and is given by $Z=(1-\partial \Sigma / \partial \omega)^{-1}[5,29]$. Next, we use the fact that in the limit $q \rightarrow 0, \omega \rightarrow 0$ the vertex function $\Gamma$ becomes

$$
\Gamma=1-\frac{\partial \Sigma}{\partial \omega}=\frac{1}{Z}
$$

This relation is an exact constraint enforcing the conservation of the total charge and is known as the first Ward identity $[30,31]$. Hence, the self-energy can be approximated as $\Sigma=$ $i G W \Gamma \approx i G_{0} W$, justifying the lack of self-consistency in the Green's function in QSGW [13]. Indeed, Kutepov confirmed that the self-consistency in $G$ and the vertex corrections in $\Sigma$ cancel out to a large extent $[32,33]$. Thus, the $Z$-factor balance is essential for accurate calculations, and its violation, as in $\Sigma=i G W$ or in $\Sigma=i G_{0} W \Gamma$, leads to an unsatisfactory accuracy for the band gaps [15,16,32]. However, the $Z$-factor cancellation was only demonstrated for the long-range limit and the remaining contribution of the vertex does not cancel out. Thus, we argue that within the QSGW approach the long-range part of the vertex can be neglected in $\Sigma$ while the short-range part has to be included to improve the QP energies.

The vertex function $\Gamma$ is highly nonlocal and even its first nontrivial term has a dependency on three spatial coordinates, making it extremely difficult to calculate explicitly. However, in the polarizability this three-point dependent contribution cancels out, which means that the vertex can be well described by an efficient two-point kernel $f_{\mathrm{xc}}$ [9]. The vertex corrections are included in the reducible polarizability $\tilde{\chi}$ through the Dyson equation as

$$
\tilde{\chi}=\chi+\chi f_{\mathrm{xc}} \tilde{\chi}
$$

The corresponding dielectric function is found through

$$
\widetilde{\varepsilon}^{-1}=1+v \tilde{\chi},
$$

and then used for screening the Coulomb interaction $\tilde{W}=$ $\widetilde{\mathcal{E}}^{-1} v$. This approach is referred to as the test charge-test charge screening [34], hereafter denoted as QSGW .

Let us assume that such an efficient two-point vertex can be expressed as the sum of a short-range and a long-range contribution:

$$
f_{\mathrm{xc}}=f_{\mathrm{xc}}^{\mathrm{LR}}+f_{\mathrm{xc}}^{\mathrm{SR}} .
$$

As discussed above, the $Z$-factor balance is required for the charge conservation, hence we need to ensure this condition in our scheme. Since the vertex is related to the xc kernel through $\Gamma=1+f_{\mathrm{xc}} \tilde{\chi}[9]$ and goes as $1 / Z$ in the long-range limit [cf. Eq. (4)], the self-energy with the two-point vertex corrections reads

$$
\begin{aligned}
\Sigma=i Z G_{0}\left(1+f_{\mathrm{xc}} \tilde{\chi}\right) \tilde{W} & =i Z G_{0}\left(1+f_{\mathrm{xc}}^{\mathrm{LR}} \tilde{\chi}+f_{\mathrm{xc}}^{\mathrm{SR}} \tilde{\chi}\right) \tilde{W} \\
& =i G_{0}\left(1+Z f_{\mathrm{xc}}^{\mathrm{SR}} \tilde{\chi}\right) \tilde{W} .
\end{aligned}
$$

In a more compact notation, the self-energy is given by

$$
\Sigma=i G_{0} \widehat{\varepsilon}^{-1} v,
$$

where $\widehat{\varepsilon}^{-1}$ includes the terms up to linear order:

$$
\widehat{\varepsilon}^{-1}=1+v \tilde{\chi}+Z f_{\mathrm{xc}}^{\mathrm{SR}} \tilde{\chi} .
$$

In Eq. (9), the long-range contribution to the vertex is canceled out and $Z f_{\mathrm{xc}}^{\mathrm{SR}}$ is the remaining part of the vertex in the short range, renormalized by $Z$. This renormalization of the vertex in the self-energy has hitherto not been considered.

Considering the formidable computational cost associated with the diagrammatic vertex corrections, a better approach for including higher-order effects in the long range is required. However, the computationally more efficient Boot approximation is based on an empirical formula and, as we show below, has a limited accuracy, especially for large-gap semiconductors. At variance, in the limit of small momenta, the Ward identity provides an exact relation between the renormalization factor $Z$ and the vertex function. The two-point vertex is connected to the $\mathrm{xc}$ kernel through the noninteracting polarizability $\chi_{0}[24]$,

$$
\Gamma=\left(1-f_{\mathrm{xc}} \chi_{0}\right)^{-1} .
$$

Since the "head" terms of the matrices $\chi_{0}$ and $f_{\mathrm{xc}}$ become dominant in the long-range limit, we can approximate them as scalar functions of momenta [36]. Thus, we use the Ward identity to derive a static xc kernel in the long-range limit,

$$
f_{\mathrm{xc}}^{\mathrm{LR}}=\frac{1-Z}{\chi_{0}^{00}},
$$


(a) Band gaps

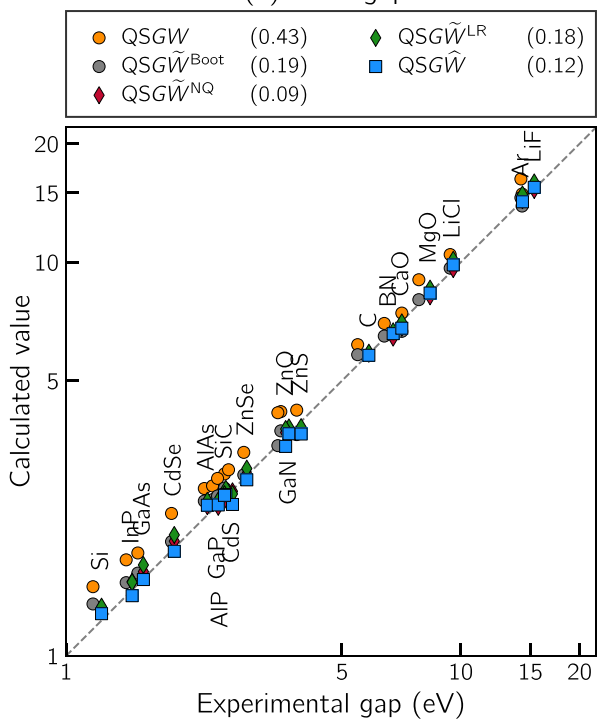

(b) Ionization potentials

\begin{tabular}{|c|c|c|c|c|c|}
\hline & PBE & $(0.92)$ & ○ & QSG $\tilde{W}^{\text {Boot }}$ & $(0.54)$ \\
\hline 0 & QSGW & $(0.65)$ & $\diamond$ & QSG $\tilde{W}^{L R}$ & $(0.51)$ \\
\hline$\diamond$ & GWГ & $(0.36)$ & $\square$ & QSGW & $(0.30)$ \\
\hline
\end{tabular}

(c) Dielectric constants

\begin{tabular}{|llll|}
\hline QSGW & $(1.37)$ & $\diamond \mathrm{QSG} \widetilde{W}^{\mathrm{LR}}$ & $(0.37)$ \\
$\diamond \mathrm{QSG} \widetilde{W}^{\mathrm{NQ}}$ & $(0.27)$ & $\square \mathrm{QSG} W$ & $(0.16)$ \\
$\circ \mathrm{QSG} \widetilde{W}^{\text {Boot }}$ & $(0.55)$ & & \\
\hline
\end{tabular}
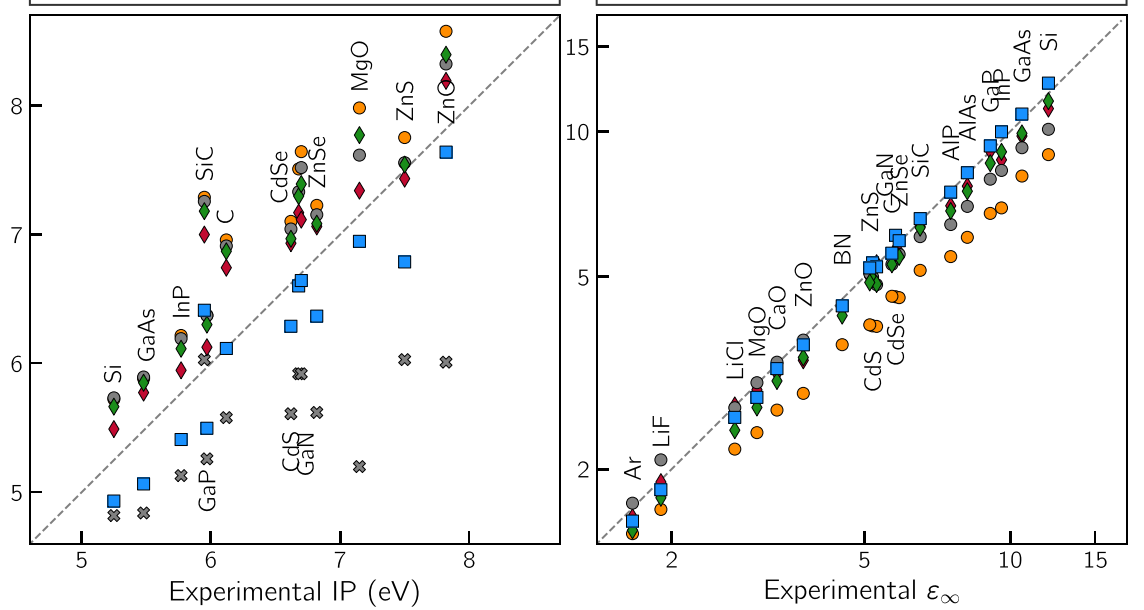

FIG. 1. Results of the QSGW calculations with various vertex corrections. The corresponding mean absolute errors (MAEs) are given in the legends (energies in eV). In (a) and (c), the band gaps and the dielectric constants are plotted on a logarithmic scale. Zero-point electron-phonon renormalizations are taken into account by modifying the experimental values [35]. In (b), the first ionization potentials obtained with Perdew-Burke-Ernzerhof (PBE) and $G W \Gamma$ are taken from Ref. [16]. The IPs are calculated as QP shifts added to the PBE values from Ref. [16]. We take the average value for the experimental IP when multiple references are available. In (c), the dielectric constants are obtained from the dielectric function defined in Eq. (6), which includes the vertex corrections.

where the superscript 00 denotes the head of the matrix. In the long-range limit, this kernel yields a vertex function that reproduces the effect of higher-order diagrams, respects charge conservation, and involves no extra cost compared to the RPA approximation. In the following, we denote this vertex function LR.

In order to assess the performance of the proposed approach, we carry out QSGEW calculations with the LR, Boot, and NQ vertex approximations. The calculations are performed for a set of semiconductors and insulators with a modified version of the code VASP [37,38] [cf. Supplemental Material (SM) [35]]. The wave functions are represented in a plane-wave basis and the ionic potential is described by the projector augmented-wave (PAW) approach [39].

In this Letter we focus on solids, but our derivation does not rely on properties specific to solids and should thus apply more generally. In the QP calculations, the renormalization $Z$ in the vertex is taken from the valence band maximum (VBM) at the $\Gamma$ point, which corresponds to the limit $q \rightarrow 0, \omega \rightarrow$ 0 . The values of $Z$ are updated self-consistently with QSGW iterations starting from 0.8 . This starting point is found to be close to the converged values and to those reported for the homogeneous electron gas [40,41].

The band gaps obtained with the various considered methods are shown in Fig. 1(a). The lack of the long-range vertex in QSGW leads to a strong overestimation of the band gaps. The LR and NQ vertices show a high accuracy with MAEs as low as 0.2 and $0.1 \mathrm{eV}$, respectively. The band gaps calculated with NQ agree with the results of Refs. [15,16]. The Boot approximation also corrects the band gaps, but it is computationally more expensive than the LR vertex as it uses an inner self-consistent loop for the vertex calculation [14]. The accuracy of the LR vertex further supports that a long-range behavior compliant with the first Ward identity is critical for correctly reproducing the band gaps.

As discussed above, the vertex in the self-energy does not have a major effect on the calculated band gaps, but is essential for obtaining accurate QP energies [21]. In the spirit of the proposed range separation, we combine our exact long-range correction with the ALDA approximation in the short-range through a Gaussian function:

$$
f_{\mathrm{xc}}^{\mathrm{LR}+\mathrm{LDA}}(k)=\frac{1-Z}{\chi_{0}^{00}} e^{-k^{2} / k_{\mathrm{TF}}^{2}}+f_{\mathrm{xc}}^{\mathrm{LDA}}\left(1-e^{-k^{2} / k_{\mathrm{TF}}^{2}}\right) .
$$

Here, $k$ is equal to $|\mathbf{q}+\mathbf{G}|, \mathbf{G}$ are reciprocal lattice vectors, and the wave vectors $\mathbf{q}$ belong to the first Brillouin zone. In this kernel, the range separation is determined by the ThomasFermi wave vector $k_{\mathrm{TF}}$, which is a characteristic screening length of the Coulomb interaction commonly used for describing the spatial variation of the dielectric function $[42,43]$. Such a simple form for the proposed vertex function can be extended to explicitly account for dynamical effects and allows for the generalization to anisotropic systems, provided the screening length and the long-range limits are properly adjusted.

In the self-energy, the LR term of the kernel cancels out, as shown in Eq. (9), leaving only the ALDA contribution. In this case, the self-energy reads

$$
\Sigma=i G_{0}\left(1+v \tilde{\chi}+Z f_{\mathrm{xc}}^{\mathrm{LDA}} \tilde{\chi}\right) v .
$$

The approach in which the vertex is included in both $\chi$ and $\Sigma$ is denoted QSGW . The details of the implementation are given in the SM [35].

This approach shows an improvement for the band gaps leading to the same accuracy as the NQ vertex corrections, 
TABLE I. Mean position (in eV) of the semicore $d$ states at the $\Gamma$ point with respect to the VBM. The QSG $\tilde{W}^{\mathrm{LR}}$ and $\mathrm{QS} G \hat{W}$ results are compared to the diagrammatic $G W \Gamma$ [16]. The calculated values are corrected to account for the effect of norm conservation (cf. SM [35]). Experimental values are taken from Ref. [16].

\begin{tabular}{lrrrr}
\hline \hline & $G W \Gamma^{\mathrm{a}}$ & $\mathrm{QS} G \tilde{W}^{\mathrm{LR}}$ & $\mathrm{QS} G \hat{W}$ & Expt. \\
\hline $\mathrm{ZnO}$ & 7.10 & 7.02 & 7.11 & 7.50 \\
$\mathrm{ZnS}$ & 8.40 & 7.95 & 8.08 & 9.00 \\
$\mathrm{ZnSe}$ & 8.60 & 8.29 & 8.42 & 9.20 \\
$\mathrm{CdS}$ & 9.50 & 9.15 & 9.41 & 9.50 \\
$\mathrm{CdSe}$ & 9.70 & 9.35 & 9.56 & 10.04 \\
$\mathrm{GaN}$ & 17.00 & 16.79 & 17.32 & 17.00 \\
$\mathrm{GaP}$ & 18.30 & 18.03 & 18.43 & 18.55 \\
$\mathrm{GaAs}$ & 18.50 & 18.10 & 18.50 & 18.80 \\
$\mathrm{InP}$ & 16.90 & 16.38 & 16.64 & 16.80 \\
$\mathrm{MAE}$ & 0.29 & 0.59 & 0.40 & \\
\hline \hline
\end{tabular}

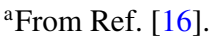

as shown in Fig. 1(a). Since the vertex corrections play an important role in the absolute QP energies, we also analyze their effect on the ionization potentials (IP). In Fig. 1(b), the IPs calculated with vertex corrections for various semiconductor surfaces are provided [44]. Without vertex corrections, QSGW strongly overestimates the IPs (MAE $0.64 \mathrm{eV}$ ) in accord with the results of Refs. [14,16]. Although the LR vertex corrections in the polarizability improve the band gaps, they barely affect the absolute band energies (MAE $0.51 \mathrm{eV}$ ). When the ALDA vertex is additionally applied to the selfenergy, as implemented in the QSGW scheme, the local screening increases and the valence band maxima shift upwards by $\sim 1 \mathrm{eV}$ on average. This results in a MAE of $0.30 \mathrm{eV}$, which is comparable to the accuracy of self-consistent diagrammatic $G W \Gamma$ [16] (MAE $0.36 \mathrm{eV}$ ). We remark that disregarding the renormalization in the short-range vertex of Eq. (14) leads to a downward shift of all the calculated IPs by about $0.2 \mathrm{eV}$, thereby strongly deteriorating the agreement with experiment. Thus, our results indicate that a high level of accuracy can be attained for both the band gaps and the IPs through the use of an efficient two-point vertex function that satisfies the $Z$-factor balance.

The considered methods can be further evaluated through their performance in describing the dielectric constants. It is known that the dielectric constants in the RPA are strongly underestimated ( $\sim 20 \%)$ and that the electron-hole interaction in the polarizability reduces the error significantly $[14,15,45,46]$. The effect of the vertex corrections on the dielectric constants is shown in Fig. 1(c). Indeed, the presence of the Boot vertex in $\chi$ reduces the error by a factor of 2 resulting in a MAE of 0.55 for QSG $\tilde{W}^{\text {Boot }}$. However, the calculated dielectric constants are still underestimated compared to both the experimental values and the QSG $\tilde{W}^{\mathrm{NQ}}$ results. The LR vertex in QSG $\tilde{W}^{\mathrm{LR}}$ shows an improved accuracy compared to the Boot approximation with a MAE of $0.37 \mathrm{eV}$. Notably, a further improvement is achieved in QSGW , where the shortrange vertex in the polarizability enhances the local screening [21], and the dielectric constants are obtained with a MAE of $0.16 \mathrm{eV}$. However, such a treatment of the local screening leads to a slight overestimation of the dielectric constants in small-gap semiconductors.

Next, we evaluate the effect of the vertex corrections on localized $d$ states. Table I shows the mean positions of the semicore $d$ bands of (post)transition metals calculated with various vertex corrections. In QSG $\tilde{W}^{\mathrm{LR}}$, the lack of vertex in the self-energy leads to the underestimation of the binding energies of the $d$ states. Indeed, the inclusion of the vertex in the self-energy in QS $G \hat{W}$ shifts the $d$ bands downwards in energy improving the agreement with experiment and making this scheme almost as accurate as $G W \Gamma$. Hence, we conclude that QSGW improves the energies of both localized and delocalized states.

The overall accuracy of the proposed QSGW approach essentially matches that of self-consistent $G W$ with secondorder diagrams for all properties considered [16]. It is important to emphasize that diagrammatic approaches are limited to small systems as their complexity increases dramatically with the order of included diagrams, whereas the present approximation does not add any extra cost to the calculations. Moreover, the proposed kernel accounts for the exact long-range asymptotic behavior and for all the local interactions through the ALDA term. Considering the success of the proposed scheme, we conclude that an approach in which higher-order terms are approximated through nondiagrammatic schemes with $Z$-factor balance provides a reliable alternative for achieving highly accurate electronic properties of solids.

In conclusion, we have developed a self-consistent QSGW approach that extends beyond $G W$ by using a range-separated vertex function that satisfies the $Z$-factor balance. We have derived the xc kernel compliant with the Ward identity in the long-range limit and proposed an approximated vertex function that spans the full spatial range. As a result, this vertex dramatically improves the quasiparticle energies of localized and delocalized states as well as the dielectric constants. Most importantly, the proposed approach achieves an accuracy similar to high-level diagrammatic $G W$ at the same cost as regular RPA calculations.

The structures and the input files used for the calculations are freely available on the Materials Cloud platform, see Ref. [47].

We thank P. Liu and G. Kresse for their help in implementing the bootstrap approximation in VASP. Support from the Swiss National Science foundation is acknowledged under Grant No. 200020-172524. The calculations have been performed at the Swiss National Supercomputing Centre (CSCS) (grant under project ID s879) and at SCITAS-EPFL.
[1] L. J. Sham and M. Schlüter, Phys. Rev. Lett. 51, 1888 (1983).

[2] J. P. Perdew and M. Levy, Phys. Rev. Lett. 51, 1884 (1983).

[3] J. P. Perdew, Int. J. Quantum Chem. 28, 497 (2009).

[4] W. Kohn, Rev. Mod. Phys. 71, 1253 (1999).
[5] L. Hedin, Phys. Rev. 139, A796 (1965).

[6] L. Hedin and S. Lundqvist, in Solid State Physics, edited by F. Seitz, D. Turnbull, and H. Ehrenreich (Elsevier, Amsterdam, 1970), Vol. 23, pp. 1-181. 
[7] M. S. Hybertsen and S. G. Louie, Phys. Rev. Lett. 55, 1418 (1985).

[8] F. Aryasetiawan and O. Gunnarsson, Rep. Prog. Phys. 61, 237 (1998).

[9] F. Bruneval, F. Sottile, V. Olevano, R. Del Sole, and L. Reining, Phys. Rev. Lett. 94, 186402 (2005).

[10] M. Gatti, F. Bruneval, V. Olevano, and L. Reining, Phys. Rev. Lett. 99, 266402 (2007).

[11] J. Vidal, F. Trani, F. Bruneval, M. A. L. Marques, and S. Botti, Phys. Rev. Lett. 104, 136401 (2010).

[12] M. van Schilfgaarde, T. Kotani, and S. Faleev, Phys. Rev. Lett. 96, 226402 (2006).

[13] T. Kotani, M. van Schilfgaarde, and S. V. Faleev, Phys. Rev. B 76, 165106 (2007).

[14] W. Chen and A. Pasquarello, Phys. Rev. B 92, 041115(R) (2015).

[15] M. Shishkin, M. Marsman, and G. Kresse, Phys. Rev. Lett. 99, 246403 (2007).

[16] A. Grüneis, G. Kresse, Y. Hinuma, and F. Oba, Phys. Rev. Lett. 112, 096401 (2014).

[17] A. M. Lewis and T. C. Berkelbach, J. Chem. Theory Comput. 15, 2925 (2019).

[18] Density Functional Theory, edited by E. K. U. Gross and R. M. Dreizler, NATO Advanced Studies Institute, Series B: Physics (Springer, Berlin, 1995), Vol. 337.

[19] Y.-M. Byun, J. Sun, and C. A. Ullrich, Electron. Struct. 2, $023002(2020)$.

[20] G. Onida, L. Reining, and A. Rubio, Rev. Mod. Phys. 74, 601 (2002)

[21] P. S. Schmidt, C. E. Patrick, and K. S. Thygesen, Phys. Rev. B 96, 205206 (2017).

[22] L. Hung, F. H. da Jornada, J. Souto-Casares, J. R. Chelikowsky, S. G. Louie, and S. Öğüt, Phys. Rev. B 94, 085125 (2016).

[23] T. Olsen, C. E. Patrick, J. E. Bates, A. Ruzsinszky, and K. S. Thygesen, npj Comput. Mater. 5, 106 (2019).

[24] R. Del Sole, L. Reining, and R. W. Godby, Phys. Rev. B 49, 8024 (1994).
[25] F. Sottile, V. Olevano, and L. Reining, Phys. Rev. Lett. 91, 056402 (2003).

[26] A. Marini, R. Del Sole, and A. Rubio, Phys. Rev. Lett. 91, 256402 (2003).

[27] G. Adragna, R. Del Sole, and A. Marini, Phys. Rev. B 68, 165108 (2003).

[28] S. Sharma, J. K. Dewhurst, A. Sanna, and E. K. U. Gross, Phys. Rev. Lett. 107, 186401 (2011).

[29] F. Aryasetiawan, L. Hedin, and K. Karlsson, Phys. Rev. Lett. 77, 2268 (1996).

[30] J. C. Ward, Phys. Rev. 78, 182 (1950).

[31] A. V. Chubukov, Phys. Rev. B 72, 085113 (2005).

[32] A. L. Kutepov, Phys. Rev. B 94, 155101 (2016).

[33] A. L. Kutepov, arXiv:1809.06654.

[34] M. S. Hybertsen and S. G. Louie, Phys. Rev. B 35, 5585 (1987).

[35] See Supplemental Material at http://link.aps.org/supplemental/ 10.1103/PhysRevB.103.L161104 for computational and implementation details.

[36] Y.-H. Kim and A. Görling, Phys. Rev. Lett. 89, 096402 (2002).

[37] G. Kresse and J. Hafner, Phys. Rev. B 47, 558 (1993).

[38] G. Kresse and J. Furthmüller, Phys. Rev. B 54, 11169 (1996).

[39] P. E. Blöchl, Phys. Rev. B 50, 17953 (1994).

[40] U. von Barth and B. Holm, Phys. Rev. B 54, 8411 (1996).

[41] B. Holm and U. von Barth, Phys. Rev. B 57, 2108 (1998).

[42] W. Chen, G. Miceli, G. M. Rignanese, and A. Pasquarello, Phys. Rev. Materials 2, 073803 (2018).

[43] Z. H. Cui, Y. C. Wang, M. Y. Zhang, X. Xu, and H. Jiang, J. Phys. Chem. Lett. 9, 2338 (2018).

[44] Z. Guo, F. Ambrosio, W. Chen, P. Gono, and A. Pasquarello, Chem. Mater. 30, 94 (2018).

[45] D. Deguchi, K. Sato, H. Kino, and T. Kotani, Jpn. J. Appl. Phys. 55, 051201 (2016).

[46] A. N. Chantis, M. van Schilfgaarde, and T. Kotani, Phys. Rev. Lett. 96, 086405 (2006).

[47] Materials Cloud Platform, 10.24435/materialscloud:hb-v3. 